\title{
Estudo dos dados obtidos na avaliação de dois glicosímetros portáteis de uso humano em cães
}

\author{
Karolliny Thais Rodrigues de Souza ${ }^{a}$, Luana Felix Monteiro ${ }^{b}$, Fabiana Batalha Knackfus ${ }^{c}$, Liliane \\ Maria Valentim Willi Monteiroc \\ aGraduanda em Medicina Veterinária na Universidade do Grande Rio, Duque de Caxias, RJ, Brasil. \\ bMédica Veterinária Autônoma no Hospital Veterinário da Universidade do Grande Rio, Duque de Caias, RJ, Brasil. \\ 'Docente do Curso de Medicina Veterinária na Universidade do Grande Rio, Duque de Caxias, RJ, Brasil.
}

RESUMO O objetivo deste estudo foi avaliar a eficiência de dois glicosímetros portáteis de uso humano em comparação ao método de referência enzimático-colorimétrico. A concentração de glicose sanguínea é um dos principais mecanismos da homeostase hormonal, sendo este um dos fatores mais preocupantes na rotina clínica veterinária. Embora os glicosímetros sejam de origem humana, tem-se mostrado com grande potencial visto sua rapidez e praticidade. Com isso, avaliá-los para a utilização na rotina veterinária torna-se imprescindível, visto que diferentes concentrações de glicose no sangue entre os seres humanos e animais podem afetar a confiabilidade e gerar resultados discrepantes ao utilizar estes aparelhos. Foram avaliadas 32 amostras sanguíneas de pacientes caninos, encaminhados da rotina clínica do Hospital Veterinário da Unigranrio em Duque de Caxias, RJ. Foram avaliados dois aparelhos (Accu-chek guide ${ }^{\circledast} e$ On call plus $\|^{\circledR}$ ), sendo utilizados testes estatísticos como D’Agostino para a normalidade dos dados; análises de medidas de tendência central; elaboração de gráficos de dispersão para visualização dos dados em comparação com o método de referência; método t (student) para dados independentes, cuja finalidade foi avaliar se houve diferença significativa entre as médias das amostras utilizadas. Também foram utilizados a normativa ISO 15197:2013 que estipula limites mais rigorosos para a avaliação de um glicosímetro, expondo a precisão do aparelho e sua eficácia. Observou que o Accu-chek guide ${ }^{\circledR}$ foi aquele que não apresentou diferenças estatísticas significativas em comparação com o método de referência e cujas variâncias foram inferiores ao glicosímetro On call plus $\|^{\oplus}$, mostrando-se um aparelho com um bom desempenho e mais preciso para utilização clínica em cães.

$$
\begin{aligned}
& \text { PALAVRAS-CHAVE: canino; diabetes; glicose sanguínea; rotina veterinária } \\
& \text { Aceito } 22 \text { de setembro de } 2021 \quad \text { Publicado online } 22 \text { de novembro de } 2021
\end{aligned}
$$

Cite este artigo: Souza et al. (2021) Estudo dos dados obtidos na avaliação de dois glicosímetros portáteis de uso humano em cães. Multidisciplinary Science Journal 3 : e2021019, doi:10.29327/multiscience.2021019.

\section{Study of data obtained in the evaluation of two portable glucometers for human use in dogs}

ABSTRACT The aim of this study was to evaluate the efficiency of two portable human-use glucometers compared to the enzymatic-colorimetric reference method. Blood glucose concentration is one of the main mechanisms of hormonal homeostasis, which is one of the most worrying factors in the veterinary clinical routine. Although glucometers are of human origin, they have shown great potential due to their speed and practicality. Therefore, evaluating them for use in the veterinary routine becomes essential, as different concentrations of glucose in the blood between humans and animals can affect reliability and generate discrepant results when using these devices. Thirty-two blood samples from canine patients referred from the clinical routine of the Unigranrio Veterinary Hospital in Duque de Caxias, RJ, were evaluated. Two devices (Accu-chek guide ${ }^{\circledast}$ and On call plus $\|^{\circledast}$ ) were evaluated, using statistical tests such as D'Agostino 
for data normality; analyzes of measures of central tendency; elaboration of scatter plots to visualize the data in comparison with the reference method; $t$ (student) method for independent data, whose purpose was to assess whether there was a significant difference between the means of the samples used. The ISO 15197:2013 standard was also used, which stipulates stricter limits for the evaluation of a glucometer, exposing the accuracy of the device and its effectiveness. It was observed that the Accu-chek guide ${ }^{\circledR}$ was the one that did not present statistically significant differences compared to the reference method and whose variances were lower than the On call plus $\|{ }^{\circledR}$ glucometer, proving to be a device with good performance and more accurate use clinic in dogs.

KEYWORDS: canine; diabetes; blood glucose; veterinary routine.

\section{Introdução}

A concentração de glicose sanguínea é um dos principais mecanismos da homeostase hormonal. Apesar de alterar o controle glicêmico de acordo com Costa e Crivellenti (2019), diversas circunstâncias podem elevar a concentração de glicose sanguínea, sendo diabetes mellitus (DM) a principal endocrinopatia. Por outro lado, a hipoglicemia é uma síndrome caracterizada pela baixa concentração de glicose plasmática, podendo ocorrer por numerosos fatores, sendo eles também por condições de erros de manejo ou portadores de doenças secundárias paralelas à DM (Costa e Crivellenti 2019).

Endocrinopatias como DM exigem constante monitoração a fim de evitar complicações (Oliveira et al 2015). A facilidade gerada pelo uso de glicosímetros (GT) na prática veterinária tornou-se permanente com o passar das décadas, facilitando no rápido acesso à glicemia assim como no controle do paciente diabético (Pöppl et al 2015). Embora os GT tenham sido inicialmente utilizados para humanos, eles têm se mostrado com grande potencial para o uso em animais, visto a rapidez e a praticidade na medicina veterinária (Stoot et al 2014). Entretanto, o consenso de diabetes para cães e gatos sugere o uso com cautela no emprego do dispositivo humano sem que os mesmos tenham sido validados para cães (Costa 2018), no entanto, o uso inadequado pode resultar em diagnóstico incorreto ou no entendimento errôneo do controle glicêmico (Nelson 2015). Os glicosímetros apresentam vários benefícios tais como, serem pequenos, portáteis e necessitarem de pouca quantidade sanguínea para medição, rapidez na obtenção dos resultados, menor custo, segurança e praticidade (Oliveira et al 2015).

Visto a importância do constante monitoramento da glicemia na rotina clínica veterinária. 0 objetivo deste estudo foi avaliar a eficiência de dois glicosímetros portáteis de uso humano em comparação ao método referência enzimático-colorimétrico.

\section{Material e métodos}

Foram avaliadas 32 amostras sanguíneas de 30 cães, atendidos no setor de endocrinologia e clínico geral do Hospital Veterinário da Universidade do Grande Rio, localizado no município de Duque de Caxias, RJ, no período de Fevereiro a Maio de 2021. Foram utilizados cães, machos e fêmeas, de raças, pesos e idades variadas num período de jejum de 12 horas, sendo eles com disfunções endócrinas, hígidos ou com outras enfermidades.

Foram coletadas amostras sanguíneas por venopunção, pelas veias jugular, cefálica ou safena, com o auxílio de agulhas $\left(25 \times 7 \mathrm{~mm}\right.$ ) ou scalp $\left(\mathrm{n}^{\circ} 23\right)$ e seringas de 3 ou $5 \mathrm{~mL}$, a fim de se obter o sangue total para fins clínicos sendo incluídas oportunamente nesta avaliação.

Aos tutores cujos pacientes participaram do estudo, foi disponibilizado o termo de consentimento antes da coleta das amostras referente aos dados descritos nesta pesquisa, sendo esta submetida para a Comissão de Ética no Uso de Animais da Unigranrio (CEUA-Unigranrio) e aprovada sobre o no de protocolo 040/2021.

Os aparelhos usados foram o Accuk-chek guide ${ }^{\circledast}$ (Figura 1) e On call plus $\|^{\circledR}$ (Figura 2) sendo eles comparados aos valores do método de referência enzimático-colorimétrico.

Após a coleta de $3 \mathrm{~mL}$ de sangue total, os mesmos foram acondicionados em frascos de $0,5 \mathrm{~mL}$ contendo etilenodiaminotetracético dissódico (EDTA) adicionado de fluoreto de sódio (glicemia); frasco de $0,5 \mathrm{~mL}$ com EDTA (Hemograma); frasco de gel com retrator de coágulo $4 \mathrm{~mL}$ (colesterol e triglicérides). O sangue total residual presente na seringa foi direcionado para mensuração imediata da glicemia nos glicosímetros portáteis. 


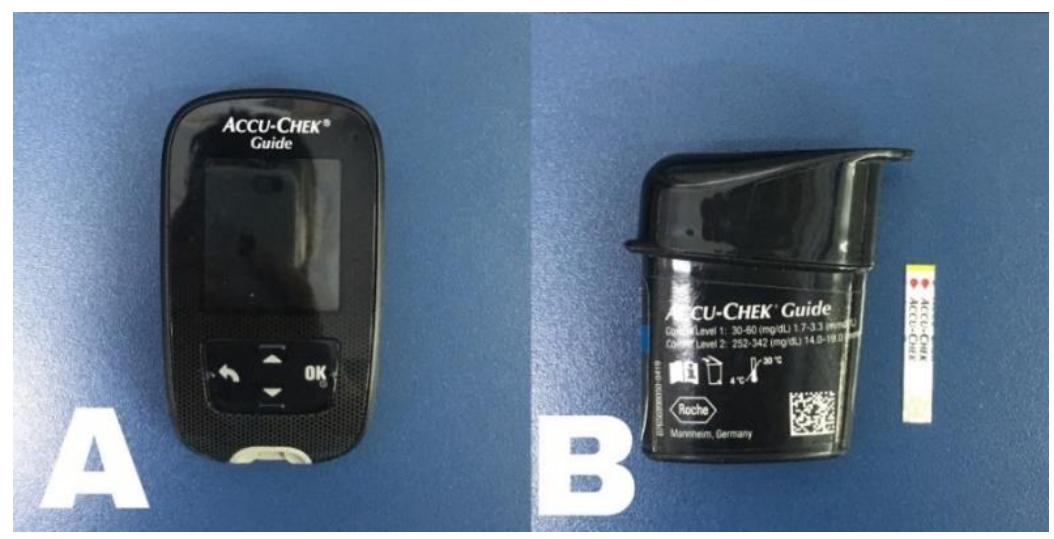

Figura 1 A - Glicosímetro portátil Accuk-chek guide ${ }^{\circledast}$. B-tira-teste.

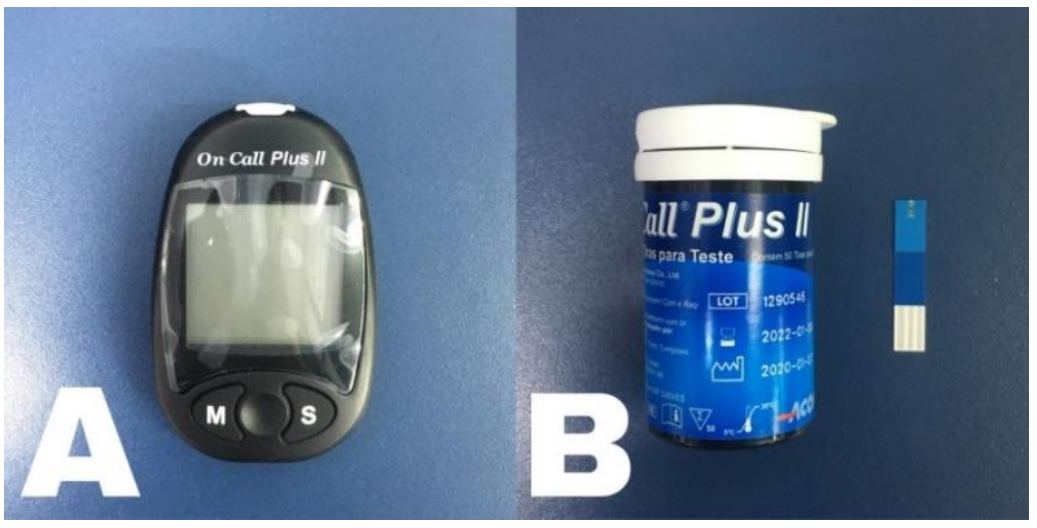

Figura 2 A - Glicosímetro portátil On call plus $\|^{\circledR}$. B-tira-teste.

Os glicosímetros usados neste estudo foram todos de uso humano, de acordo com as orientações do fabricante, o aparelho accu-chek guide ${ }^{\circledR}$, não necessitou fazer a calibração após as trocas de lote de tiras teste (Figura 1B), pois o dispositivo já se mostrava apto para aceitar uma nova fita a cada troca. Já o on call plus $/{ }^{\circledR}$ foi calibrado com soluçãocontrole disponível pelo mesmo, usado sempre após a abertura de um novo frasco de tiras-teste (Figura 2B), ambos manuseados de acordo com as recomendações de seus fabricantes, as tiras-teste de glicemia utilizadas no trabalho foram de lotes diferentes, todas dentro do prazo de validade.

Os aparelhos utilizados apresentam parâmetros diferentes entre si, os quais podem ser observados resumidamente no Tabela 1 e na Tabela 2 indicam à faixa dos parâmetros que interferem nos resultados obtidos pelos aparelhos.

Tabela 1 Parâmetros dos glicosímetros de uso humano avaliados nesta pesquisa, com relação ao método de ensaio, tipo da amostra, método de determinação do valor de referência, faixa de medição do sistema (miligramas por decilitro), volume da amostra (microlitro) e duração do teste (segundos) dos respectivos aparelhos e suas funcionalidades de acordo com o protocolo dos seus fabricantes.

\begin{tabular}{|c|c|c|c|c|c|c|}
\hline Glicosímetros & $\begin{array}{l}\text { Método de } \\
\text { ensaio }\end{array}$ & $\begin{array}{l}\text { Tipo da } \\
\text { Amostra }\end{array}$ & $\begin{array}{c}\text { Método de } \\
\text { determinação do } \\
\text { valor de referência }\end{array}$ & $\begin{array}{c}\text { Faixa de } \\
\text { medição do } \\
\text { sistema(mg/dL) }\end{array}$ & $\begin{array}{c}\text { Volume da } \\
\text { Amostra } \\
(\mu \mathrm{L})\end{array}$ & $\begin{array}{l}\text { Duração do } \\
\text { teste } \\
\text { (segundos) }\end{array}$ \\
\hline $\begin{array}{l}\text { Accu-chek } \\
\text { Guide }^{\circledR}\end{array}$ & Amperometria & $\begin{array}{c}\text { Sangue } \\
\text { capilar e total }\end{array}$ & $\begin{array}{c}\text { Método } \\
\text { hexoquinase }\end{array}$ & $10-600$ & 0,6 & $<4$ \\
\hline On call plus $\|^{\circledR}$ & Coulometria & $\begin{array}{c}\text { Sangue } \\
\text { capilar e total }\end{array}$ & Glicose oxidase & $20-600$ & 1,0 & 4 \\
\hline
\end{tabular}

O sangue total contido nos seus respectivos tubos foi destinado ao laboratório de análises clínicas do próprio hospital da Universidade do Grande Rio, onde foi realizada a análise laboratorial das amostras, sendo classificadas segundo as concentrações de glicose, medida pelo método de referência (60 - 100 mg/dL). 
Tabela 2 Parâmetros de limitações dos glicosímetros utilizados, que podem influenciar nos valores obtidos de acordo com os critérios de seus respectivos fabricantes.

\begin{tabular}{ccc}
\hline Glicosímetro & Accu-chek guide & On call plus II \\
\hline Hematócrito $(\%)$ & $10-65$ & $30-55$ \\
Triglicérides $(\mathrm{mg} / \mathrm{dL})$ & $>1.800$ & $<3.000$ \\
Colesterol $(\mathrm{mg} / \mathrm{dL})$ & Não esclarecido & $<500$ \\
Ácido úrico $(\mathrm{mg} / \mathrm{dL})$ & Não esclarecido & $\leq 20$ \\
Ácido ascórbico $(\mathrm{mg} / \mathrm{dL})$ & Não esclarecido & $<3$ \\
Bilirrubina $(\mathrm{mg} / \mathrm{dL})$ & Não esclarecido & $\leq 40$ \\
Teste de absorção de xilose & Não esclarecido & Não esclarecido \\
\hline
\end{tabular}

O método referência usado para a obtenção dos valores glicêmicos modelo foi o método enzimáticocolorimétrico (Trinder), que tem como finalidade, reagentes com destino a determinar a somatória da glicose no sangue, considerando a glicose oxidase (GOD) a qual catalisa a oxidação da glicose para ácido glucónico e peróxido de hidrogênio. A leitura das amostras foi realizada utilizando kits comerciais da Biotécnica ${ }^{\circledR}$ e analisador bioquímico automatizado.

Quanto a análise estatística, foi aplicado o teste D’Agostino para verificar a normalidade dos dados e posteriormente utilizou-se o teste $\mathrm{t}$ de (student) para análise dos dados independentes, tendo sido realizadas as comparações do accu-chek guide ${ }^{\circledR}$ e do on call plus $\|^{\circledR}$ utilizando-se o método de referência.

As medidas de tendência central como média, mediana além do mínimo, máximo, desvio padrão e variação, foram estimadas para as variáveis quantitativas, sendo avaliados os dois glicosímetros com o método de referência. Igualmente, também foram avaliados os hematócritos, triglicerídeos e colesterol.

Para melhor visualização dos dados, foram elaborados gráficos de dispersão linear e boxplot, demonstrando os glicosímetros utilizados com o método de referência. Para todas as análises foi considerado $5 \%$ de probabilidade de erro, sendo utilizado o programa BioEstat 5.0.

Analisaram-se os glicosímetros e o método de referência, mediante as normativas ISO 15197:2013. Segundo as recomendações da ISO 15197:2013 que estabelecem limites de precisão mais rigorosos. Ela determina que 95\% dos valores variem até $\pm 15 \mathrm{mg} / \mathrm{dL}$ da referência quando a glicose é $<100 \mathrm{mg} / \mathrm{dL}$, e dentro de $\pm 15 \%$ quando a glicose é $\geq 100$ $\mathrm{mg} / \mathrm{dL}$.

\section{Resultados}

Das 32 amostras utilizadas, foram incluídas neste estudo 28 para a comparação das glicemias utilizando dois glicosímetros portáteis. Foram excluídos quatro animais em função das suas glicemias se apresentarem acima de 277 $\mathrm{mg} / \mathrm{dL}$ pelos glicosímetros e pelo método de referência.

De acordo com a clínica dos animais avaliados, foram incluídos pacientes com diagnóstico de endocrinopatias como: hipotireoidismo, diabetes mellitus, hipoadrenocorticismo (Addison) e hiperadrenocorticismo (HAC), sendo também observados pacientes hígidos, com neoplasias ou encaminhados para o pré-cirúrgico. Todos os animais portadores de endocrinopatias e neoplasias já se apresentavam sob terapia de controle.

Com relação ao gênero e a idade dos pacientes, foram incluídos 16 fêmeas e 12 machos, com idades variando entre 1 e 17 anos. Separados em GTA (accu-chek guide ${ }^{\circledR}$ ) e GTB (on call plus // ${ }^{\circledR}$ ), as amostras sanguíneas contidas nesta pesquisa demostraram seus valores de hematócrito no intervalo de 28 a 64\%, triglicerídeos até $490 \mathrm{mg} / \mathrm{dL}$ e colesterol até $438 \mathrm{mg} / \mathrm{dL}$ (Tabela 3). Estes valores estão dentro dos parâmetros de referência preconizados de acordo com as limitações dos aparelhos utilizados (Tabela 2).

Nos glicosímetros portáteis, os resultados das amostras inseridas foram entre 71 (mínima) a $269 \mathrm{mg} / \mathrm{dL}$ (máxima), correspondente ao GTA e de 50 a $213 \mathrm{mg} / \mathrm{dL}$ correspondente ao GTB. Separadas de acordo com a sua classificação fisiológica (Tabela 4), foram classificados como hipoglicemia valores abaixo da referência $(<60 \mathrm{mg} / \mathrm{dL})$; hiperglicemia acima (>100 mg/dL) e normoglicemia dentro dos valores de referência de normalidade estabelecidos pelo laboratório utilizado (60 - $100 \mathrm{mg} / \mathrm{dL})$. 
Tabela 3 Valores de Hematócrito, Triglicerídeos e Colesterol dos animais inclusos neste estudo, de acordo com as limitações preconizados pelos seus fabricantes.

\begin{tabular}{|c|c|c|c|}
\hline \multicolumn{4}{|c|}{ Amostras inclusas } \\
\hline Animais & Hematócrito (\%) & Triglicerídeos (mg/dL) & Colesterol (mg/dL) \\
\hline 1 & 50 & 161 & 136 \\
\hline 2 & 38 & 136 & 348 \\
\hline 3 & 44 & 155 & 208 \\
\hline 4 & 43 & 83 & 180 \\
\hline 5 & 60 & 334 & 318 \\
\hline 6 & 50 & 114 & 285 \\
\hline 7 & 43 & 490 & 310 \\
\hline 8 & 41 & 44 & 130 \\
\hline 9 & 48 & 47 & 221 \\
\hline 10 & 53 & 83 & 180 \\
\hline 11 & 53 & 60 & 438 \\
\hline 12 & 43 & 73 & 166 \\
\hline 13 & 30 & 95 & 311 \\
\hline 14 & 55 & 65 & 156 \\
\hline 15 & 35 & 194 & 266 \\
\hline 16 & 37 & 131 & 155 \\
\hline 17 & 50 & 45 & 193 \\
\hline 18 & 40 & 139 & 294 \\
\hline 19 & 50 & 121 & 188 \\
\hline 20 & 47 & 149 & 204 \\
\hline 21 & 34 & 40 & 230 \\
\hline 22 & 44 & 59 & 241 \\
\hline 23 & 53 & 170 & 303 \\
\hline 24 & 49 & 51 & 167 \\
\hline 25 & 50 & 356 & 288 \\
\hline 26 & 64 & 74 & 252 \\
\hline 27 & 46 & 67 & 289 \\
\hline 28 & 28 & 67 & 178 \\
\hline
\end{tabular}

Tabela 4 Número de amostras de pacientes com relação à glicemia, separadas de acordo com a classificação fisiológica, tipo de glicosímetro e método de referência.

\begin{tabular}{cccc}
\hline & \multicolumn{4}{c}{ Número de amostras } \\
\hline Classificação Fisiológica & Accu-chek guide & On call plus II & Método de referência \\
\hline Hipoglicemia & 0 & 11 & 0 \\
Normoglicemia & 25 & 13 & 13 \\
Hiperglicemia & 3 & 4 & 15 \\
Total & 28 & 28 & 28 \\
\hline Nota - Hipoglicemia: valores abaixo da referência $(<60 \mathrm{mg} / \mathrm{dL}) ;$ Normoglicemia: dentro dos valores referência de \\
normalidade (60 - $100 \mathrm{mg} / \mathrm{dL}) ;$ Hiperglicemia: acima do valor de referência $(>100 \mathrm{mg} / \mathrm{dL})$.
\end{tabular}

Através das análises estatísticas, foi possível observar que não houve diferença significativa entre o glicosímetro accu-chek guide ${ }^{\circledast}(p>0,05)$ em comparação com o método de referência. Entretanto, foi possível observar diferença 
estatística entre o glicosímetro on call plus $\|{ }^{\circledast}(p<0,05)$ em comparação ao método de referência (Tabela 5 e Figura 3 ), demonstrando que, as médias de glicemia por meio desses dois aparelhos diferiram entre si.

Os dois glicosímetros avaliados apresentaram resultados de glicemia menores em comparação com o método de referência. No entanto, observou-se que os resultados gerados pelo aparelho accu-chek guide ${ }^{\circledR}$ apresentou uma menor variação dos dados. A média glicêmica pelo método de referência foi de $105 \mathrm{mg} / \mathrm{dL}$ e notou-se que no glicosímetro accu-chek guide ${ }^{\circledR}$ foi de $95 \mathrm{mg} / \mathrm{dL}$ e no on call plus $\|^{\otimes} 77 \mathrm{mg} / \mathrm{dL}$ (Tabela 6).

Tabela 5 Média, Mínimo, Máximo, Desvio padrão (DP), Primeiro e Terceiro quartis e coeficiente de variação dos valores de glicemias em miligramas por decilitro (mg/dL), observados em relação ao tipo de glicosímetro avaliado.

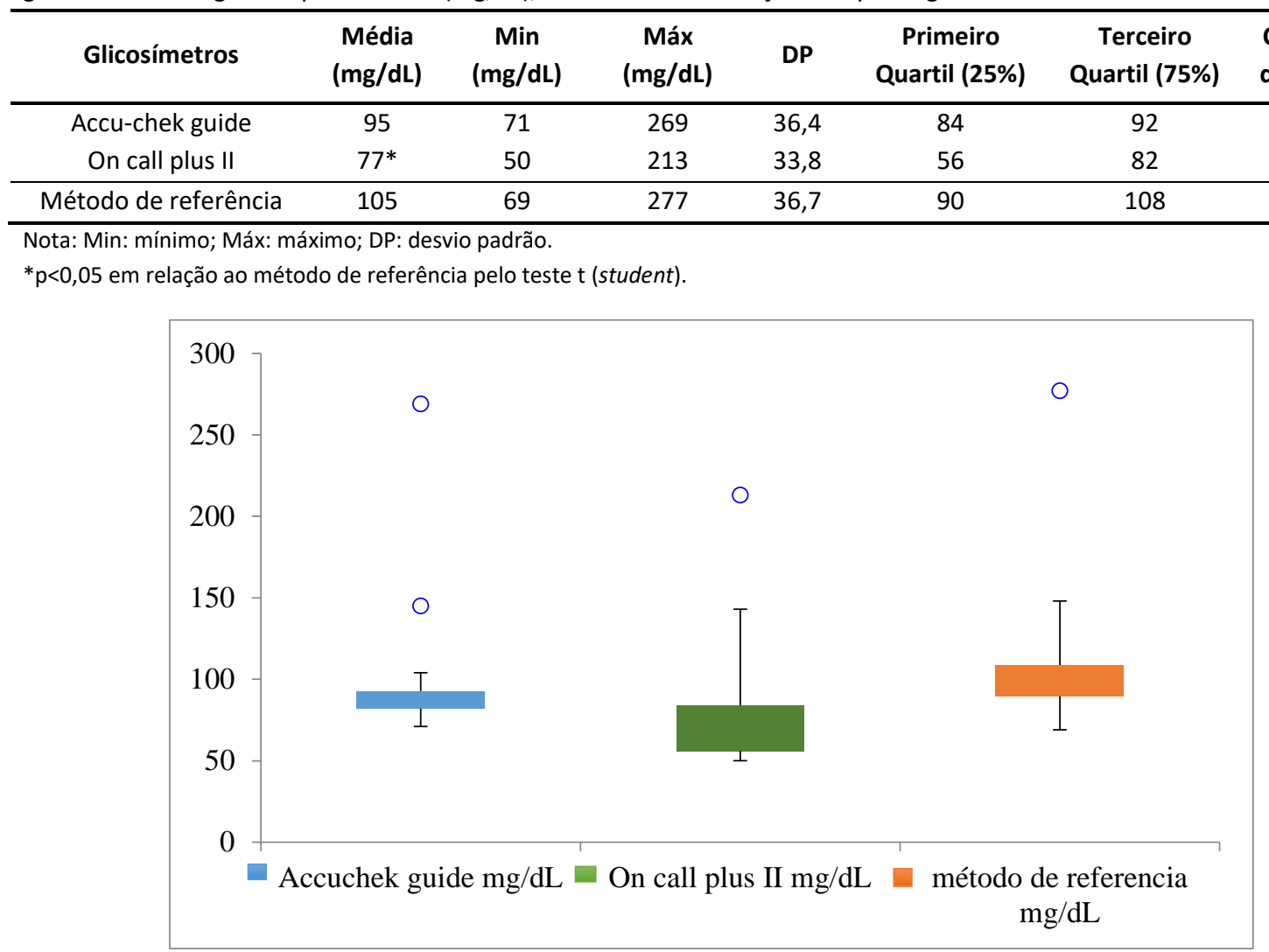

Figura 3 Boxsplot da distribuição dos valores de glicemias em relação aos glicosímetros avaliados no presente estudo, sendo azul accu-chek guide $^{\circledast}$, verde on call plus ${ }^{\circledast}$ e laranja método de referência. As linhas medianas presentes nas caixas representam a média dentro de cada avaliação, as barras representam os limites inferiores e superiores, o achatamento do box fornece a variação do dado e os outliers encontram-se representados pelos círculos na cor azul.

Os dois aparelhos avaliados apresentaram correlações positivas quando comparados com o método de referência, no entanto, o glicosímetro accu-chek guide ${ }^{\circledR}$ evidenciou uma maior correlação, sendo esta a mais próxima de 1 (Tabela 6). Os resultados realizados pelos aparelhos apresentaram coeficiente positivo, demonstrando que maiores valores pelo método de referência corresponderam a uma tendência de crescimento em ambos os aparelhos.

Nas análises das Figuras 4 e 5, foram observados que resultados acima de $140 \mathrm{mg} / \mathrm{dL}$, houve uma maior dispersão dos dados quando comparados com o método de referência. Entretanto, observou-se que no glicosímetro on call plus II ${ }^{\circledR}$ (Figura 5) foi superior.

De acordo com normativa da ISO 15197: 2013, nenhum dos dois aparelhos avaliados alcançaram todas as recomendações, as quais que preconizam $95 \%$ dos resultados variem até $\pm 15 \mathrm{mg} / \mathrm{dL}$ da referência quando a glicose é até $99 \mathrm{mg} / \mathrm{dL}$, e dentro de $\pm 15 \%$ quando a glicose é $\geq 100 \mathrm{mg} / \mathrm{dL}$. No entanto, o aparelho accu-chek guide ${ }^{\circledR}$ apresentou um melhor desempenho, atingindo $86 \%$ dos resultados nas faixas glicêmicas determinadas. Já os resultados atingidos pelo aparelho on call plus $/{ }^{\circledast}{ }^{\circledR}$ foi de apenas $25 \%$ dos resultados exigidos pela normativa ISO 15197: 2013. 
Tabela 6 Coeficiente de correlação dos dois glicosímetros utilizados neste estudo, demonstrando sua correlação com o método de referência

\begin{tabular}{ccc}
\hline Glicosímetros & Coeficiente de correlação & P-valor \\
\hline Accu-chek guide & 0,985 & $<0.0001$ \\
On call plus II & 0,932 & $<0.0001$ \\
\hline
\end{tabular}

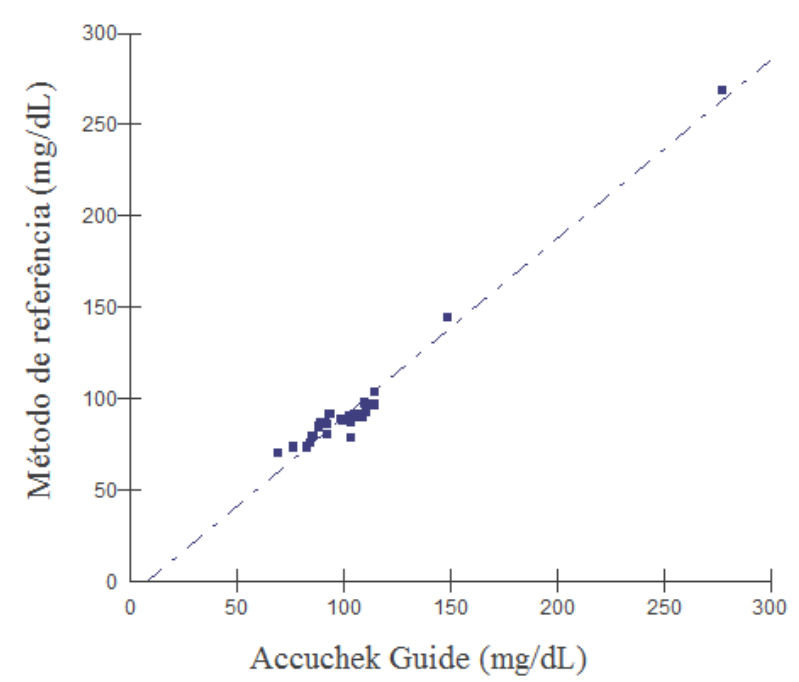

Figura 4 Gráfico de dispersão linear das glicemias avaliadas pelo glicosímetro accu-chek guide ${ }^{\circledast}$ em comparação como método de referência.

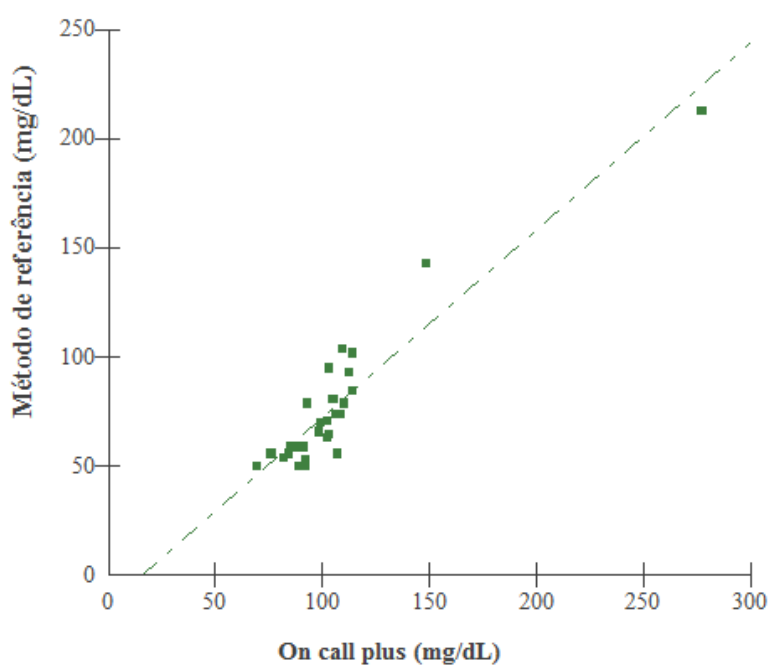

Figura 5 Gráfico de dispersão linear das glicemias avaliadas pelo glicosímetro on call plus $/ /{ }^{\circledR}$ em comparação como método de referência.

\section{Discussão}

De acordo com os resultados obtidos foi observado que os dois glicosímetros apresentaram menores valores de glicemia em relação ao método de referência. Cohen et al. (2009) e Nelson (2015) citam que resultados glicêmicos obtidos pela maioria dos glicosímetros portáteis geralmente apresentam resultados inferiores quando comparados com o método de referência, e que a diferença tende a ser maior quando há hiperglicemia. Inclusive, neste estudo, foram excluídas cinco amostras cujas glicemias se apresentaram com valores discrepantes, corroborando com os resultados apresentados por estes autores.

Corrêa (2017) esclarece que, apesar da faixa de hematócrito se apresentarem dentro da faixa preconizada pelos aparelhos para avaliação do seu desempenho, na rotina clínica, essa faixa pode ser ultrapassada de acordo com a 
variação do sangue total. Essa variação é mais susceptível em animais portadores de DM não controlados, uma vez que o sintoma apresentado pelos animais com esta clinica pode levar a uma desidratação, gerando uma hemoconcentração e alterando o valor real do hematócrito. Sendo assim, neste estudo, o fato do glicosímetro accu-chek guide ${ }^{\circledR}$ apresentar um intervalo de hematócrito maior que o aparelho on call plus $\|^{\circledR}$, atribui a ele um melhor desempenho na rotina.

Segundo as limitações dos aparelhos utilizados, as faixas de triglicerídeos e colesterol apresentaram-se dentro dos parâmetros preconizados pelos glicosímetros. Porém, Santos (2020) cita que a elevação de triglicerídeos e colesterol no plasma podem ser causadas por causas primárias ou secundárias, sendo as secundárias principalmente em animais com endocrinopatias, como diabetes mellitus, hiperadrenocorticismo e hipotireoidismo, ou as primárias como animais da raça schnauzer entre outras circunstâncias, essas faixas podem ser ultrapassadas. Os animais avaliados no presente estudo, todos estavam sendo acompanhados pelo médico veterinário, sendo assim a avaliação periódica do paciente é de extrema importância antes do emprego dos glicosímetros, a fim de prevenir que qualquer interferência ocorra nos valores de glicemia por questões bioquímicas e, além disso, manter a saúde e o bem estar do animal.

Este trabalho permitiu a confirmação de que o uso do glicosímetro na prática veterinária torna-se muito eficaz. Devido sua rapidez no decorrer da pesquisa e a obtenção do valor das glicemias de forma rápida e prática, foram ótimas ao ser comparado com a demora da realização do exame laboratorial usado como referência. Isso mostra que em uma situação de emergência, usá-los traria benefícios à saúde do paciente e para uma conduta certa do quadro clinico apresentado pelo paciente.

Segundo Pöppl et al. (2015), o lançamento de novos aparelhos no mercado ocorre com maior frequência devido a evolução dos estudos baseado em pacientes diabéticos, tornando importante a avaliação deles antes do uso na rotina veterinária. Sendo este aparelho de uso humano ou animal, Cohen et al. (2009) cita que nem todos, mesmo usados amplamente na medicina veterinária, se mostram aptos para tal, da mesma forma que até mesmo os glicosímetros desenvolvidos para uso animal podem não apresentar resultados confiáveis. Esta informação corrobora com o propósito deste estudo, reforçando que a avaliação estatística e clínica dos aparelhos são de suma importância, para que possam ser utilizados na rotina veterinária, pois o uso de aparelhos humanos não avaliados pode levar a diagnósticos errôneos ao serem utilizados em cães.

\section{Conclusões}

Portanto, o presente estudo demonstra que o glicosímetro accu-chek guide ${ }^{\circledR}$ não apresentou diferença nos resultados obtidos quanto ao método de referência, podendo ser observados tanto pelas médias aferidas, quanto pelo coeficiente de correlação. Contudo, segundo a ISO 15197:2013, nenhum dos dois aparelhos alcançaram os padrões preconizados pela normativa, porém o accu-chek guide ${ }^{\circledR}$ apresentou um melhor desempenho sobre o on call plus // ${ }^{\circledR}$. Com isso, foi possível concluir que o glicosímetro portátil accu-chek guide ${ }^{\circledR}$ foi o que mais se aproximou do método de referência, sugerindo que esse é o método mais acurado para aferir a glicemia em pacientes caninos na rotina veterinária.

\section{Conflito de Interesses}

Não há conflito de interesses entre os autores.

\section{Financiamento}

Esta pesquisa não recebeu auxílio financeiro

\section{Referências}

Cohen TA, Richard WN, Philip HK, Mary MC, Edward CF (2009) Evaluation of six portable blood glucose meters for measuring blood glucose concentration in dogs. Journal of the American Veterinary Medical Association. DOI: 10.2460/javma.235.3.276

Corrêa ML (2017) Avaliação de dois glicosímetros veterinários para uso em cães. Dissertação, Universidade Federal de Minas Gerais.

Costa PB, Crivellenti SB (2019) Homeostase glicêmica: Mecanismos moleculares, consequências fisiológicas e métodos de mensuração da glicemia em cães e gatos, clinica medica de pequenos animais. DOI: 10.26843/investigacaov1812019p\%25p 
Costa PB (2018) Mucosa labial como sitio alternativo para monitoração glicêmica transcirúrgica de cães e gatos saudáveis. Dissertação, Universidade Federal de Uberlândia.

ISO 15197:2013, In vitro diagnostische testsystemen - Eisen voor bloedglucose - monitoring systemen voor zelftesten ten behoeve van het reguleren van diabetes mellitus (ISO 15197:2013).

Nelson RW (2015) Canine Diabetes Mellitus. In: Feldman, E C Nelson R W (ed) Canine and Feline Endocrinology and Reproduction, $4^{\mathrm{a}}$ ed. W B Saunders Company, Phildelphia, pp 213-314.

Oliveira YSGO, Junior JDC, Santos-Leonardo A, Morais KS (2015) Comparação entre os métodos laboratorial e portátil na análise da glicemia em felinos com amostras de sangue venoso central e capilar. Ciência Animal Brasileira. DOI: 10.1590/1089-6891v16i231266

Pöppl ÁG, Valle SF, Bianchi SP, Moresco MB, Carvalho GC (2015) Avaliação do desempenho de três glicosímetros portáteis para mensuração de glicemia em cães: um estudo-piloto. Veterinary \& Science 9: 37-40.

Santos KSN (2020) Interferência da hemólise, lipemia e icterícia nos exames bioquímicos. Dissertação, Universidade Federal do Rio Grande do Sul.

Stoot LJ, Cairns NA, Cull F, Taylor JJ, Jeffrey JD, Morin F, Mandelman J, Clark TD, Cooke SJ (2014) Use of portable blood physiology point-of-care devices for basic and applied research on vertebrates: a review. Conservation Physiology. DOI: 10.1093/conphys/cou011 